\title{
Continuous neonatal blood gas monitoring using a multiparameter intra-arterial sensor
}

\author{
C Morgan, S J Newell, D A Ducker, J Hodgkinson, D K White, C J Morley, J M Church
}

Neonatal Intensive Care Unit, St James's University Hospital, Leeds

C Morgan

S J Newell

All Saint's Hospital, Chatham, Kent

D A Ducker

J Hodgkinson

Rosie Maternity Unit, Addenbrooke's

Hospital, Cambridge

D K White

C J Morley

Diametrics Medical Ltd, High Wycombe, Bucks

J M Church

Correspondence to: Dr S J Newell,

Neonatal Intensive Care

Unit,

St James's University

Hospital,

Leeds LS9 $7 \mathrm{TF}$.

Accepted 16 October 1998

\begin{abstract}
Aims-To compare arterial blood gas (ABG) readings obtained with a multiparameter intra-arterial sensor with those from an ABG analyser.

Methods-An MPIAS with the ability to measure continuously $\mathbf{p H}, \mathrm{PaCO}_{2}$, and $\mathrm{PaO}_{2}$ was introduced via an umbilical arterial catheter in 27 neonates requiring intensive care. They underwent 3260 hours of MPIAS monitoring, during which 753 ABG readings were performed.

Results-Overall bias (mean difference: MPIAS-ABG) and precision (standard deviation of differences) values were: -0.002 and 0.022 , respectively, for $\mathbf{p H}$; +0.26 and 0.52 for $\mathrm{PaCO}_{2}(\mathrm{kPa})$; and -0.19 and 0.99 for $\mathrm{PaO}_{2}(\mathrm{kPa})$. This gave $95 \%$ limits of agreement as: $\mathbf{- 0 . 0 4 7}$ to $+\mathbf{0 . 0 4 2}$ for $\mathrm{pH},-0.76$ to $+1.28 \mathrm{kPa}$ for $\mathrm{PaCO}_{2}$, and -2.13 to $+1.75 \mathrm{kPa}$ for $\mathrm{PaO}_{2}$. For each variable, precision across readings from the same individual was better than overall precision for all data. No complications related to the use of the catheter were observed.
\end{abstract}

Conclusions-Continuous MPIAS ABG monitoring is an exciting development, with the potential to reduce blood transfusions and improve ABG homeostasis. (Arch Dis Child Fetal Neonatal Ed 1999;80:F93-F98)

Keywords: blood gas monitoring; multiparameter intraarterial sensor

Blood gas monitoring is an essential part of neonatal intensive care, its primary aim being to maintain arterial blood gas homeostasis, with particular emphasis on avoiding large peturbations. Currently this is achieved using information obtained from intermittent arterial blood gas (ABG) analysis. This is still the

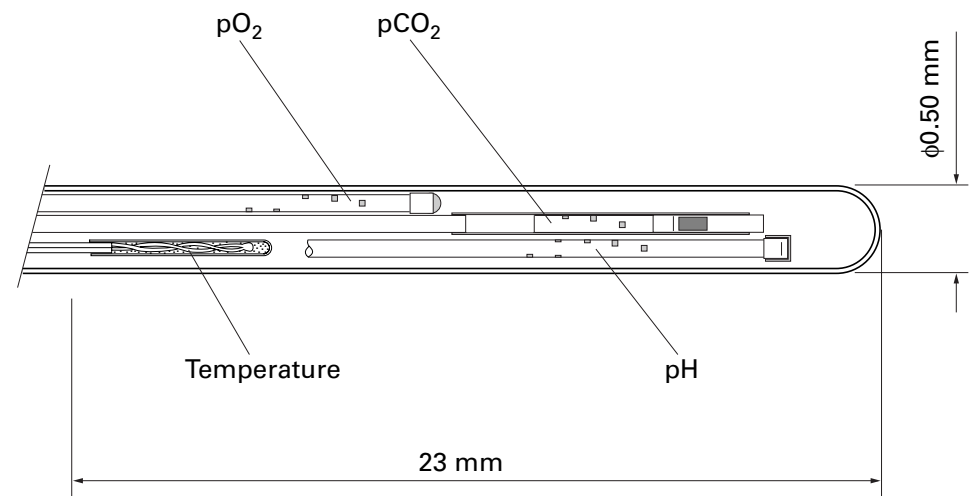

Figure 1 Schematic of distal 23 mm of MPIAS, showing location of individual $p H$, $\mathrm{PaCO}_{2}$, and $\mathrm{PaO}_{2}$ sensors and thermocouple. gold standard in terms of accuracy and precision. Clinical usefulness is limited because ABG analysis is performed intermittently, with a frequency determined by either clinical condition or local neonatal intensive care practice. It is well known that even in stable intensive care patients sudden variations can occur. There are, therefore, potential clinical benefits in continuous $\mathrm{ABG}$ monitoring.

The need for continuous ABG monitoring has been addressed by the introduction of a number of non-invasive, indirect methods. In particular, transcutaneous $\mathrm{CO}_{2}$ and pulse oximetry are now part of routine neonatal intensive care monitoring. Unfortunately, transcutaneous monitoring requires frequent resiting and/or recalibration of the probe and is least reliable in the sickest infants. ${ }^{1}$ The limitations of information from pulse oximetry have been well described. ${ }^{23}$

Continuous intra-arterial $\mathrm{PaO}_{2}$ monitoring has been possible for more than two decades using an umbilical arterial catheter with a Clark electrode. ${ }^{4}$ These catheters are highly reliable, although accuracy does deteriorate with deposition of blood and protein on the catheter tip. ${ }^{4}$ More recently, continuous monitoring of intra-arterial $\mathrm{pH}, \mathrm{PaCO}_{2}$, and $\mathrm{PaO}_{2}$ in paediatric and adult intensive care patients has become available ${ }^{67}$ using a multiparameter intra-arterial sensor (MPIAS), placed in a peripheral artery. An MPIAS has now been developed using new technology that allows it to be passed through an umbilical arterial catheter. We report the first evaluation of the Neotrend (Diametrics Medical Ltd, High Wycombe, UK) in newborn infants receiving intensive care.

We aimed to: compare the measurements of $\mathrm{pH}, \mathrm{pCO}_{2}$, and $\mathrm{pO}_{2}$ from the MPIAS with those from the blood gas analyser; calculate bias and precision for each variable for the whole group and individual patients; and characterise the type, incidence, and severity of complications associated with the use of the Neotrend MPIAS.

\section{Methods}

The Neotrend MPIAS has three optical sensors made of $175 \mu \mathrm{m}$ polymethylmethacrylate fibres with radial holes drilled through the fibres (at the tip) to allow the appropriate indicators to be placed in the optical pathway (fig 1). A signal light beam is sent down the optic fibres. A stainless steel mirror is encapsulated within the end of each fibre. This reflects the altered light beam back along the optic fibre to the detectors. This arrangement effectively means that each fibre represents a miniature spectrophotometer. 
The design of the individual sensors is now described in brief. The holes in the $\mathrm{pH}$ sensor are filled with polyacrylamide gel containing immobilised phenol red dye. The dye changes colour (reversibly) in response to the concentration of hydrogen ions. By measuring the absorbency of green light by the red dye, the $\mathrm{pH}$ can be measured. The $\mathrm{PaCO}_{2}$ sensor is similar. The holes in the $\mathrm{PaCO}_{2}$ sensor are enclosed within a gas only permeable membrane. Encapsulated within, is a bicarbonate solution containing phenol red dye as an indicator. $\mathrm{CO}_{2}$ diffuses across the membrane and alters the $\mathrm{pH}$ of the solution. The $\mathrm{PaO}_{2}$ sensor contains an entrapped fluorescent ruthenium based dye in a silicon rubber matrix. Blue light is absorbed by the fluorescent dye and emitted as light of a different wavelength. The emitted light intensity is altered by the presence of oxygen, allowing the $\mathrm{PaO}_{2}$ to be calculated. The three individual sensors are assembled with a T-type thermocouple to form the combined sensor bundle of the MPIAS (fig 1). All four components are contained within a microporous polyethylene outer sheath. The pores in the surface are filled with polyacrylamide gel, continuous with the gel of the individual sensors. The outer surface of the sheath is coated with covalently bound active heparin. The outer diameter of the MPIAS is $0.5 \mathrm{~mm}$.

The MPIAS is sealed within a device to allow its introduction into the umbilical arterial catheter lumen. The Sensor Introduction System (SIS) consists of a sliding mechanism to introduce the sensor in and out of a sealed cable. At the distal end is a Y-connector. An "O-ring" seal, through which the sensor is introduced, prevents bleeding back when connecting to the umbilical arterial catheter. Improvement in the SIS and arterial catheter design continued during the study. SIS from five different batches were used in this group of patients. Each batch was modified according to clinical experience with the previous batch. These changes were implemented to facilitate clinical use and improve the method used to pass the sensor down the lumen of the umbilical arterial catheter. No modifications were made to the MPIAS itself. The problems encountered related to non-uniformity of umbilical arterial catheter length and difficulty in threading the sensor through the O-ring (resulting in sensor damage, usually to the thermocouple). The rate of successful MPIAS insertion was considerably improved in subsequent batches.

The calibration procedure is automated and lasts 35 minutes. Each MPIAS is provided with its tip in a tonometer containing buffer solution. During calibration, sterile mixtures of gases with known concentrations of $\mathrm{CO}_{2}$ and $\mathrm{O}_{2}$ are passed through the solution. With simultaneous measurement of the barometric pressure, the concentration of $\mathrm{CO}_{2}$ and $\mathrm{O}_{2}$, as well as $\mathrm{pH}$, can be changed. The signals from the individual sensors are recorded and used to generate equations that predict the response of the sensor signals to changes in analyte concentration. These are stored within the patient data module.
The MPIAS and calibrated module are transferred to the display monitor at the cotside. The MPIAS is then connected to, and passed down, the umbilical arterial catheter. The tip of the sensor lies $23 \mathrm{~mm}$ distal to the catheter tip. This ensures that the active tip of all four individual sensor components sit in arterial blood within the lumen of the descending aorta. The clinical monitor graphically displays the change in blood gases together with aortic temperature continuously over time. Current blood gas status is digitally displayed along with temperature and calculated values for base excess, bicarbonate and oxygen saturation. The monitor can be adjusted to show up to 24 hours of data.

Local hospital ethics committee approval was obtained at all centres. Patients were recruited at St James's University Hospital, Leeds, All Saint's Hospital, Chatham, and Addenbrooke's Hospital, Cambridge. Informed consent was obtained before sensor placement. All patients admitted to neonatal intensive care and requiring an umbilical arterial catheter were eligible for study. The study was designed as a prospective trial with sequential patient enrolment. Only patients with more than 24 hours of continuous monitoring and over 10 complete datasets were eligible for analysis. A complete dataset consists of $\mathrm{pH}, \mathrm{PaCO}_{2}$, and $\mathrm{PaO}_{2}$.

Umbilical arterial catheters $(4 \mathrm{~F})$ were placed at the level T10. The Neotrend MPIAS was then calibrated and inserted. Time was then allowed for the MPIAS to adapt from reading the calibration solution to displaying stable ABG values (less than 5 minutes). Stable ABG was confirmed by the presence of tick symbols above each digital display before collecting data. It was established that all four components of the MPIAS tip were distal to the catheter tip and reading ABG (and not umbilical arterial catheter flush), by sampling the catheter. Displayed values should remain stable during ABG sampling. Each time an ABG sample was taken for analysis, a hard copy of the current Neotrend MPIAS clinical display was printed. The ABG samples were analysed using a Radiometer 510 (SJUH), Ciba Corning $565(\mathrm{ASH})$, or Chiron Diagnostics $865(\mathrm{AH})$ blood gas analysers. The ABG and Neotrend printouts were then collected. Complete datasets $\left(\mathrm{pH}, \mathrm{PaCO}_{2}\right.$, and $\left.\mathrm{PaO}_{2}\right)$ for both methods at each sampling time were retained for analysis.

The indwelling MPIAS can be recalibrated on the basis of any ABG result. Such calibrations were kept to a minimum. Recalibrations only took place where there was either a large offset in one or more variables from insertion, or there was evidence of progressive and consistent drift over the previous 24 hours. All such recalibrations were documented. The study was concluded in each patient when either the umbilical arterial catheter was no longer required clinically or when removal was clinically indicated. The reasons for removal were recorded.

The terms bias and precision are used throughout, and are defined as: 
(A) $\mathrm{pH}$
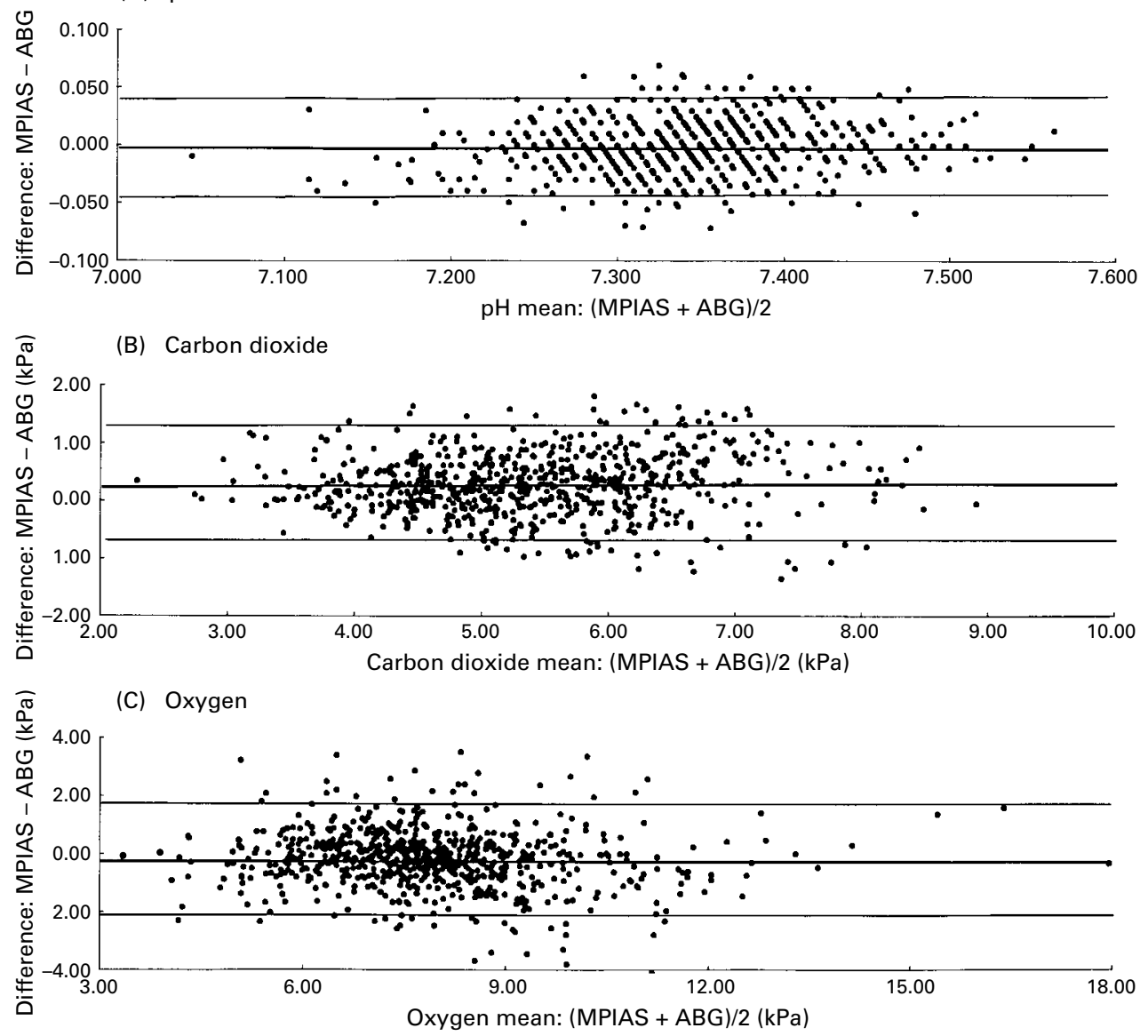

Figure 2 Bland and Altman plots for combined data, for each variable. Overall bias is shown as bold horizontal line. $95 \%$ upper and lower limits of agreement are shown as thinner horizontal lines.

- bias: mean difference between MPIAS and ABG readings (for each variable)

- precision: standard deviation of the differences between MPIAS and ABG readings.

Bland and Altman plots ${ }^{8}$ were prepared for each variable, for combined patient data. This enabled calculation of overall bias (mean difference: MPIAS-ABG) and precision (standard deviation of differences). Individual patient data were analysed using bias/precision plots of individual patients for each variable.

\section{Results}

Data were analysed from all 27 patients (14 girls) successfully monitored in the three study centres. Median (range) birthweight and gestation were 1170 (560-3200) g and 28 (24-40) weeks, respectively. Median (range) time for MPIAS monitoring was 93.9 (24.1-428.8) hours. Nine patients were monitored for more than 7 days. Median (range) of complete datasets per patient was $25(12-75)$. There was a total of 3260 hours of monitoring time, generating 753 complete datasets. Bland and Altman analyses $^{8}$ were performed on the data from all patients. Bland and Altman plots are shown for $\mathrm{pH}$ (fig 2A), $\mathrm{PaCO}_{2}$ (fig 2B), and $\mathrm{PaO}_{2}$ (fig 2C). Overall bias (mean difference: MPIAS$\mathrm{ABG}$ ) and precision (standard deviation of differences) for $\mathrm{pH}, \mathrm{PaCO}_{2}$, and $\mathrm{PaO}_{2}$ are shown in table 1 .
Table 1 Overall bias (mean difference: MPIAS-ABG) and precision (standard deviation of differences) values for each variable; LLA: $95 \%$ lower limit of agreement. ULA:95\% upper limit of agreement

\begin{tabular}{lllll}
\hline$N=753$ & Bias & Precision & LLA & ULA \\
\hline $\mathrm{pH}$ & -0.002 & 0.022 & -0.047 & +0.041 \\
$\mathrm{PaCO}_{2}(\mathrm{kPa})$ & +0.26 & 0.52 & -0.76 & +1.28 \\
$\mathrm{PaO}_{2}(\mathrm{kPa})$ & -0.19 & 0.99 & -2.13 & +1.75 \\
\hline
\end{tabular}

There was a tendency for the MPIAS to over read the $\mathrm{PaCO}_{2}$. Indeed, the bias was positive in 23 out of 27 patients, although the range of mean bias for individual patients was -0.3 to $+1.0 \mathrm{kPa}$. This wide range in bias is apparent for the other variables and has an important effect on interpreting overall precision values when data from different patients are combined. This is of particular significance in continuous ABG monitoring, where bias can be reduced or eliminated by recalibration against a true $A B G$ and trend analysis is clinically important. To address this difficulty in interpreting the overall precision figure, we have produced bias/precision data for individual patients. These are shown for $\mathrm{pH}$ (fig 3A), $\mathrm{PaCO}_{2}$ (fig 3B), and $\mathrm{PaO}_{2}$ (fig 3C). For each variable, the precision value for most individual patients is below that of the overall precision figure: $\mathrm{pH}$ (23 patients), $\mathrm{PaCO}_{2}$ (24 patients), and $\mathrm{PaO}_{2}$ (19 patients). This clearly shows that precision is much better within individual 
(A) $\mathrm{pH}$

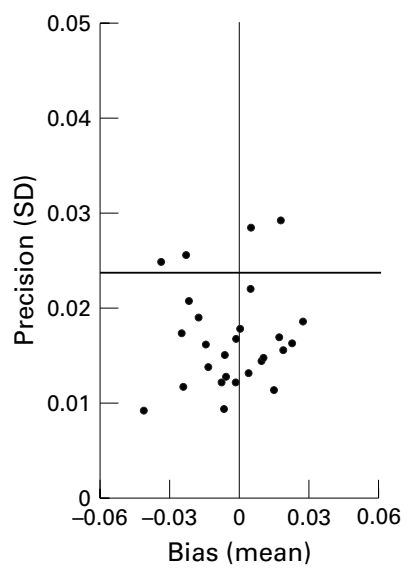

(B) Carbon dioxide

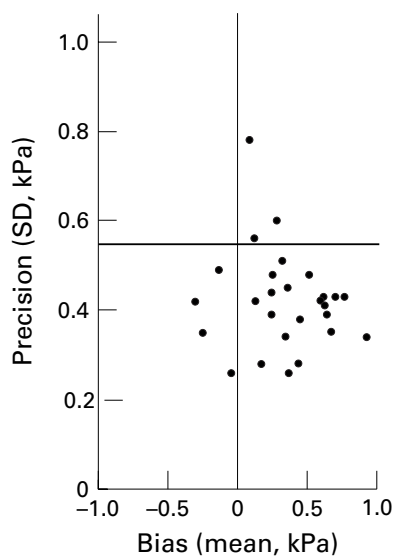

(C) Oxygen

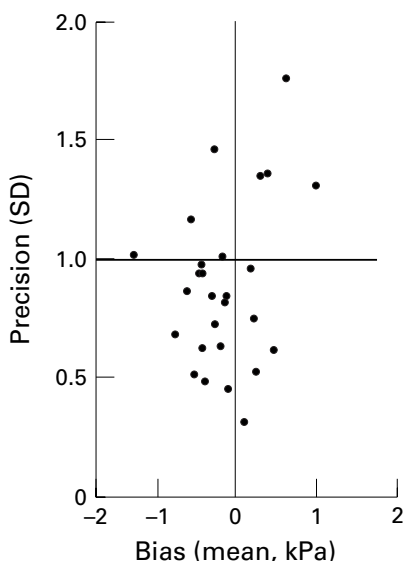

Figure 3 Bias/precision plots for 27 patients, for each variable. Overall precision (1 SD) for each variable is shown as bold horizontal line.

patients than in the overall data, for all three measurements.

Recalibrations were kept to a minimum: 17 patients did not require any recalibration. The variables recalibrated in the remaining 10 patients were: $\mathrm{pH}$ (four patients), $\mathrm{PaCO}_{2}$ (five patients), and $\mathrm{PaO}_{2}$ (eight patients). All were single recalibrations. Thus a total of 17 recalibrations (any variable) were made over 3280 hours of monitoring. This indicates that there was a minimal tendency for the MPIAS to drift with time. The most common scenarios leading to a need for calibration were: a drift down in $\mathrm{PaO}_{2}$ over the first 24 hours (then stopping) or a very slow drift up in $\mathrm{PaCO}_{2}$ after 5 to 7 days of monitoring. Clearly, these were not consistent findings and affected only a few patients.

Any adverse events during monitoring were recorded, as were the reasons for removal of the umbilical arterial catheter. No problems with blood pressure transducer monitoring were observed and there was no damping of the trace with the current SIS design. In 16 patients the catheter was removed because it was no longer required; in eight the catheter stopped sampling. Two patients died because of severe lung disease. The remaining patient had the catheter removed after 25 hours because of haematuria. This was related to Escherichia coli septicaemia, disseminated intravascular coagulation (DIC), and a catheter that had migrated from $\mathrm{T} 10$ to $\mathrm{L} 1$ on $x$-ray picture. Renal and Doppler ultrasonography remained normal and the patient made a complete recovery. One patient ( 24 weeks gestation, 770 g) had an extremely difficult delivery, with cord prolapse and the need for rotation before assisted breech delivery. Examination of the placenta revealed a large retro-placental clot. At delivery there was a severe metabolic acidosis. She was very ill with evidence of a coagulopathy. This infant developed signs of paraplegia. Magnetic resonance imaging revealed three areas of spinal cord haemorrhage between the mid thoracic and lumbar regions consistent with trauma rather than vascular accident. This was confirmed by an independent neurological and neuroradiological opinion.

During the trial, a further 19 patients underwent attempts to insert an MPIAS. The rate of successful placement was specifically not an aim of this study, because it was anticipated that the SIS would require modification when clinical use started. We have reported it because it is clearly clinically important. In 11 patients the insertion was either unsuccessful $(n=8)$ or resulted in an incorrectly placed sensor $(n=3)$. As indicated in the methods, this did not result from difficulties in passing the MPIAS down the lumen of the umbilical arterial catheter, but related to difficulties with the seal mechanism (O ring) between the catheter and the SIS. The proportion of successful insertions increased as the SIS was modified. In the remaining eight patients the catheter had to be removed before generating enough data (all underwent two or more ABG samples). This group included patients who developed transient lower limb duskiness $(n=2)$, or who had catheters that stopped sampling $(n=2)$, or were no longer required (4 samples). No other complications were seen in this group.

\section{Discussion}

There are several reasons why continuous ABG monitoring in neonatal intensive care may be clinically beneficial. The effects of changes in $\mathrm{PaCO}_{2}$ on cerebral blood flow in preterm infants have been well described. ${ }^{9}$ Both hypercapnia and hypocarbia are associated with the development of intraventricular haemorrhage and periventricular leucomalacia. ${ }^{10-13}$ Therefore, in preterm infants $\mathrm{PaCO}_{2}$ has to be kept within strict limits, particularly in the first 72 hours of intensive care. The need to avoid hyperoxia as well as hypoxia has similar implications for $\mathrm{PaO}_{2}$. Moreover, rapid changes in ABG can occur after surfactant administration, especially natural surfactants, ${ }^{14}$ and during high frequency oscillatory ventilation. Indeed, some workers have suggested patients receiving the latter should always have continuous $\mathrm{PCO}_{2}$ monitoring. ${ }^{15}$ 
The Neotrend MPIAS provides $\mathrm{pH}$ sensor readings that agree closely with those of $\mathrm{ABG}$ analysis, with negligible bias and good precision. The $\mathrm{PaCO}_{2}$ sensor showed positive bias in most individuals with acceptable precision. Finally, the $\mathrm{PaO}_{2}$ sensor showed an inconsistent bias with acceptable precision. The fourth variable, aortic temperature, was not studied, as there is no standard for comparison available, although it is a potentially useful clinical indicator in sepsis. The biases shown for individual sensors are similar to the pattern reported for the Paratrend MPIAS in adult ${ }^{16} 17$ and paediatric ${ }^{7}$ intensive care patients. However, the precision is better than demonstrated in most of these studies, particularly for $\mathrm{PaO}_{2}$. This is partly because the earlier Paratrend sensor monitored the $\mathrm{PaO}_{2}$ with a Clark electrode. Furthermore, there are difficulties with peripheral arterial lines because of artefacts resulting from limb movement and changing blood flow. ${ }^{16}$ The neonatal descending aorta does not create these difficulties.

It is also important to consider the precision of ABG analysers. A recent study ${ }^{18}$ found negligible variation within instruments, where precision (SD of the differences) values of 0.03 and $0.06 \mathrm{kPa}$ were reported for $\mathrm{PaCO}_{2}$ and $\mathrm{PaO}_{2}$, respectively. Comparison of 10 different analysers gave precision values of 0.13 and $0.18 \mathrm{kPa}$ for $\mathrm{PaCO}_{2}$ and $\mathrm{PaO}_{2}$, respectively, although the full clinical range of $\mathrm{PaCO}_{2}$ and $\mathrm{PaO}_{2}$ values was not tested. Other comparisons between two $A B G$ analysers, using the whole ABG range, have shown less precision, with $0.011,0.18$, and $2.8 \mathrm{kPa}$ reported for $\mathrm{pH}$, $\mathrm{PaCO}_{2}$, and $\mathrm{PaO}_{2}$, respectively. ${ }^{19}$ These figures are similar to those found for portable ABG analysers, although $\mathrm{PaCO}_{2}$ precision varied from $0.26-0.48 \mathrm{kPa}$ in recent studies. ${ }^{19-21}$

Clearly, it is useful to consider the variation between two ABG analysers (two gold standards) when interpreting the ABG analyser/ Neotrend MPIAS precision figures. The level of overall precision for the Neotrend MPIAS is not sufficient to eliminate the need for ABG sampling. Nevertheless, the precision of the MPIAS will considerably reduce the frequency of ABG sampling and therefore the need for blood transfusion. Our clinical experience also suggests that MPIAS monitoring facilitates earlier detection of endotracheal tube obstruction and pneumothorax than current monitoring methods. Similarly, MPIAS monitoring seemed to have beneficial effects on the speed of ventilator weaning and the avoidance/ reduction of periods of hypo/hypercapnia. Such anecdotal findings point to the need for controlled trials to assess both the potential short and long term benefits.

MPIAS monitoring also has to be considered in the light of non-invasive alternative techniques. Clearly, non-invasive methods of continuous monitoring are still appropriate in infants in whom umbilical arterial catheter insertion is unsuccessful or not clinically indicated. However, MPIAS monitoring is a highly attractive option in those who do merit a catheter. Intra-arterial $\mathrm{PaO}_{2}$ monitoring via the Clark electrode is well established in neonatal intensive care. ${ }^{4}$ Precision of the Clark electrode has not been subject to Bland and Altman analysis. The reported correlation coefficients are similar to that of the MPIAS, particularly if an allowance for the Clark electrode response time is made when it is calibrated. ${ }^{22}$ Moreover, the use of a fluorescence sensor instead of a Clark electrode for $\mathrm{PaO}_{2}$ monitoring, seems to avoid the drift with time, associated with the latter. ${ }^{5}$

With intra-arterial $\mathrm{PaO}_{2}$ monitoring already available, the justification for MPIAS monitoring has to come from monitoring the other variables. This essentially asks: how well does $\mathrm{PaCO}_{2}$ monitoring compare with transcutaneous $\mathrm{PCO}_{2}\left(\mathrm{TcPCO}_{2}\right)$ monitoring? This comparison is not straightforward. The initial validation studies for $\mathrm{TcPCO}_{2}$ monitoring were performed before the limitations of using correlation coefficients for such purposes were evident. ${ }^{8}$ These studies indicated a positive bias (over reading) and reasonable precision. ${ }^{23} 24$ While it was suggested that a correction factor could be used, ${ }^{23}$ it became apparent that the error increased with higher $\mathrm{PaCO}_{2}{ }^{25}$ and electrode temperature. ${ }^{26}$ Some studies also experienced large drifts. ${ }^{27}{ }^{28}$ Most importantly, $\mathrm{TcPCO}_{2}$ monitoring seemed to be least reliable in the sickest patients, particularly in the presence of hypotension. ${ }^{1}$ More recently, the validation of $\mathrm{TcPCO}_{2}$ has been reappraised using the methods of Bland and Altman. Carter $e t a l^{29}$ reviewed two newer devices, demonstrating bias (precision) values $(\mathrm{kPa})$ of $+0.05(0.33)$ and $-0.13(0.47)$. These values seem impressive, but both groups lost patients to analysis, either as outliers or because of excessive drift. Moreover, while over a quarter of the patients received inotropes, the demographic details (median birthweight $2.3 \mathrm{~kg}$, gestation 34 weeks) are not those of the sickest intensive care infants. The greatest disadvantage of $\mathrm{TcPCO}_{2}$ monitoring, however, remains the need to recalibrate and resite at least 4 hourly, more so in the smallest infants. ${ }^{30}$ The MPIAS demonstrated minimal drift and so rarely needed recalibration. MPIAS monitoring avoids excess handling, loss of monitoring time and retains precision, even in sick infants.

As well as providing an advantageous alternative to current continuous $\mathrm{PaO}_{2}$ and $\mathrm{PaCO}_{2}$ techniques, the MPIAS has two novel features. It permits continuous arterial $\mathrm{pH}$ monitoring. This advance has the potential to provide early warning of deteriorations in the metabolic component of arterial blood gas status. The method of introducing the MPIAS after umbilical arterial catheter placement also allows the clinician to defer MPIAS insertion. Thus in those infants in whom it is not clear whether continuous intra-arterial blood gas monitoring is justified, the decision can be deferred (for the first few hours of intensive care) without losing the opportunity to establish umbilical arterial access.

It is important to understand that the purpose of this study was to validate the sensor (MPIAS) in vivo and not the mechanism used to introduce it (SIS). The SIS was modified five times during the study and this 
substantially improved the success rate of insertion. Thus the failure rate in this study does not reflect the reliability of the current design. In those infants in whom the catheter had to be removed "before clinically indicated," seven out of eight had been in place for more than 7 days. We did not find evidence of thrombus formation in the catheter tips. We did not find any complications associated with using the MPIAS. The complications following the breech delivery were unusual, but so is the combination of tractional and rotational forces on the spinal column in association with intrapartum asphyxia in a preterm infant. We were sufficiently concerned to arrange for an independent opinion which confirmed that this was not an event associated with use of the catheter.

In conclusion, the Neotrend MPIAS represents an exciting development in the field of continuous monitoring in neonatal intensive care. Bias/precision data indicate that it is a valuable clinical tool. It will not eliminate the need for ABG sampling, but the potential benefits of reduced blood transfusions and earlier intervention in adverse events are clear. Whether this translates into long term patient benefits, needs to be examined in randomised controlled trials.

We thank Selina-Jane Walker and the neonatal intensive care staff in all three units for their support during the study. We are grateful to Diametrics Medical Ltd for their contribution to the funding of this project.

Brunstler I, Enders A, Versmold HT. Skin surface PCO monitoring in new born infants in shock: effect of hypoten-
sion and electrode temperature. 7 Pediatr 1982;100:454-7.

sion and electrode temperature. F Pediatr 1982;100:454-7.
2 Dear PRF. Monitoring oxygen in the newborn. Arch Dis Child 1987;62:879-81.

3 Jennis MS, Peabody JL. Pulse oximetry: an alternative method for the assessment of oxygenation in newborn infants. Pediatrics 1987;79:524-8.

4 Goddard P, Keith I, Markovich H, Roberton NRC, Rolfe P, Scopes JW. The use of a continuously recording intravascular oxygen electrode in the newborn. Arch Dis Child 1974;49:853-60

5 Pollitzer MJ, Soutter LP, Reynolds EOR. Continuous monitoring of arterial oxygen tensionin infants: four year experience with an intravascular oxygen electrode. Pediat rics 1980;66:31-6.

6 Venkatesh B, Clutton-brock TH, Hendry SP. Continuous measurement of blood gases using a combined electrochemical and spectrophotometric sensor. F Med Eng Techchemical and spect

7 Weiss IK, Fink S, Edmunds S, Harrison R, Donnelly K. Continuous arterial gas monitoring: initial experience with
Paratrend 7 in children. Intensive Care Med 1996;22:14147 .
8 Bland JM, Altman DG. Statistical methods for assessing agreement between two methods of clinical measurement. Lancet 1986;i:307-10.

9 Leahy FAN, Cotes D, MacCallum M, Rigatto LI. Effect of $\mathrm{CO}_{2}$ and 100 per cent $\mathrm{O}_{2}$ on cerebral blood flow in preterm infants. F Appl Physiol 1980;48:468-72.

10 Cooke RW. Factors associated with periventricular haemorrhage in very low birthweight infants. Arch Dis Child 1981;56:425-31.

11 Levene MI, Fawer C-L, Lamont RF. Risk factors in the development of periventricular haemorrhage in the preterm neonate. Arch Dis Child 1982;57:410-17.

12 Greisen G, Munck H, Lou H. Severe hypocarbia in preterm infants and neurodevelopmental deficit. Acta Paediatr Scand 1987;76:401-4.

13 Ikonen RS, Janas MO, Koivikko MJ, Laippala P, Kuusinen EJ. Hyperbilirubinaemia, hypocarbia and periventricular leukomalacia in preterm infants: relationship to cerebral palsy. Acta Paediatr Scand 1992;81:802-7.

14 Kresch MJ, Lin WH, Thrall RS. Surfactant replacement therapy. Thorax 1996;51:1137-54.

15 Chan V, Greenough A. The effect of frequency on carbon dioxide levels during high frequency oscillation. $\mathcal{f}$ Perinat Med 1994:22:103-6.

16 Venkatesh B, Clutton-Brock TH, Hendry SP. A multivariable sensor for continuous intra-arterial monitoring: a prospective evaluation. Crit Care Med 1994;24:588-94

17 Zollinger A, Spahn DR, Singer T, et al. Accuracy and clinical performance of a continuous intra-arterial blood gas monitoring system during thoracoscopic surgery. $\mathrm{Br} f$ Anaesth 1997;79:47-52.

18 Kampelmacher MJ, van Kesteren RG, Winckers EK. Instrumental variability of respiratory blood gases among different blood gas analysers in different laboratories. Eur Respir F 1997;10:1341-4.

19 Wahr JA, Lau W, Tremper KK, Hallock L, Smith K. Accuracy and precision of a new portable, handheld blood gas analyzer, the IRMA 7 Clin Monitor 1996;12:317-24.

20 Zagola GP, Roberts PR, Black K, Santamauro JT, Klase E, Suleiman $M$. Hand held blood gas analyzer is accurate in the critical care setting. Crit Care Med 1996;24:957-62.

21 Mahutte CK, Holody M, Maxwell TP, Chen PA, Sasse SA. Development of a patient dedicated, on-demand, blood gas monitor. Am 7 Resp Crit Care Med 1994;149:852-9.

22 Wilkinson AR, Phibbs $\mathrm{RH}$, Gregory GA. Improved accuracy of continuous measurement of arterial oxygen in sick newborn infants. Arch Dis Child 1979;54:307-10.

23 Hansen TN, Tooley WH. Skin surface carbon dioxide tension in sick infants. Pediatrics 1979;64:942-7.

24 Cassady G. Transcutaneous monitoring in the newborn infant. F Pediatr 1983;103:837-48.

25 Martin RJ, Beoglos A, Miller MJ, et al. Increasing arterial carbon dioxide tension: influence on transcutaneous carbon dioxide tension measurements. Pediatrics 1988;81:684-7.

26 Wimberley PD, Frederikson PJ, Witt-Hansen J, et al. Evaluation of a transcutaneous oxygen and carbon dioxide ation of a transcutaneous oxygen and ca
monitor. Acta Paediatr Scand 1985;74:352-9.

27 Whitehead MD, Halsall D, Pollitzer MJ. Transcutaneous estimation of arterial $\mathrm{PO}_{2}$ and $\mathrm{PCO}_{2}$ in newborn infants with a single electrochemical sensor. Lancet 1980;i:111114

28 Kost GJ, Chow JL, Kenny MA. Monitoring transcutaneous carbon dioxide tension. Am f Clin Pathol 1983;80:832-8.

29 Carter B, Hochmann M, Osborne A, Nisbet A, Campbell $\mathrm{N}$. A comparison of two transcutaneous monitors for the measurement of arterial $\mathrm{PO}_{2}$ and $\mathrm{PCO}_{2}$ in neonates. Anaesth Intensive Care 1995;23:708-14.

30 Rennie J. Trancutaneous carbon dioxide monitoring. Arch Dis Child 1990;65:345-6. 